\title{
Impact of cataract on the spectral measurement of fundus autofluorescence
}

\author{
Rowena Simon ${ }^{1} \cdot$ Jakob Lauritz Brauer ${ }^{1} \cdot$ Daniel Meller ${ }^{1} \cdot$ Martin Hammer $^{1,2}(\mathbb{C}$
}

Received: 4 November 2021 / Revised: 30 December 2021 / Accepted: 4 January 2022 / Published online: 12 January 2022

(c) The Author(s) 2022

\section{Key message}

- Fundus autofluorescence emission spectra are known to be specific for lipofuscin and other fluorophores.

- However, fluorescence is green-shifted by the lens in case of cataract.

\section{Dear Editor,}

Fundus autofluorescence (FAF) can arise from a variety of fluorophores. To distinguish those fluorophores, FAF can be characterized by its fluorescence lifetime and by its emission spectrum. The spectral characteristic of FAF can give valuable information on pathologic changes in RPE and accumulation of metabolic byproducts in age-related macular degeneration [1,2]. Recently, we introduced the peak emission wavelength (PEW), calculated from the ratio of fluorescence intensities in the two spectral channels of the fluorescence lifetime imaging ophthalmoscope (FLIO), as an estimate measure of the emission spectrum [3]. Here, we investigate the influence of cataract on the PEW.

We performed FLIO measurements of FAF in 32 eyes of 32 patients (age: $72.3 \pm 9.3$ years) scheduled for cataract extraction. The study followed the tenets of the Declaration of Helsinki and was approved by a local ethics committee. Informed consent was obtained from all subjects before inclusion. FLIO investigations were performed one day before and on average $33.2( \pm 10.3)$

Martin Hammer

martin.hammer@med.uni-jena.de

1 Department of Ophthalmology, University Hospital Jena, Am Klinikum 1, 07747 Jena, Germany

2 Center for Medical Optics and Photonics, Univ. of Jena, Jena, Germany days after cataract extraction. FLIO counts single fluorescence photons with respect to their time delay relative to the excitation laser pulse $(470 \mathrm{~nm})$ in two spectral channels (500-560 $\mathrm{nm}$ and 560-720 nm). Here we disregard the temporal information and calculate the PEW from the ratio of fluorescence photons per pixel in the two spectral channels as described earlier [3]. PEW were averaged over standardized areas at the fundus by centering the standard ETDRS grid at the fovea. The foveal PEW was calculated as mean value of pixels within the center of the grid and the value for the posterior pole as average over the pixels in the inner and outer ring of the grid.

Figure 1 shows the color-coded PEW for one subject before (left) and after (right) cataract extraction; a red-shift is clearly seen. Averaged over all subjects, the PEWs were $523 \pm 29 \mathrm{~nm}$ and $592 \pm 17 \mathrm{~nm}$ before as well as $599 \pm 18 \mathrm{~nm}$ and $608 \pm 11 \mathrm{~nm}$ after cataract extraction in the fovea and at the posterior pole, respectively (Fig. 2). No difference between nuclear $(N=12)$ and cortical $(N=20)$ cataract was found for the pre-operative PEW. The pre- and post-operative values were different with high significance $(p<0.001$ in the t-test in both areas) and the effect was strong (Cohen's $\mathrm{d}=1.416$ for fovea and 0.723 for the posterior pole).

This indicates that FAF observations, even if using confocal scanning techniques, are overlaid by the lens fluorescence. This way, our current study corroborates similar observations with respect to fluorescence lifetime [4]. As the human lens has a fluorescence emission maximum at 
Fig. 1 Color-coded PEW (see scale bar) of a patient before (left) and after (right) cataract extraction. In the right-hand panel, the ETDRS grid is shown

Fig. 2 Histogram of PEW distributions at the posterior pole (top) and fovea (bottom) over all patients $(n=32)$ before and after cataract extraction
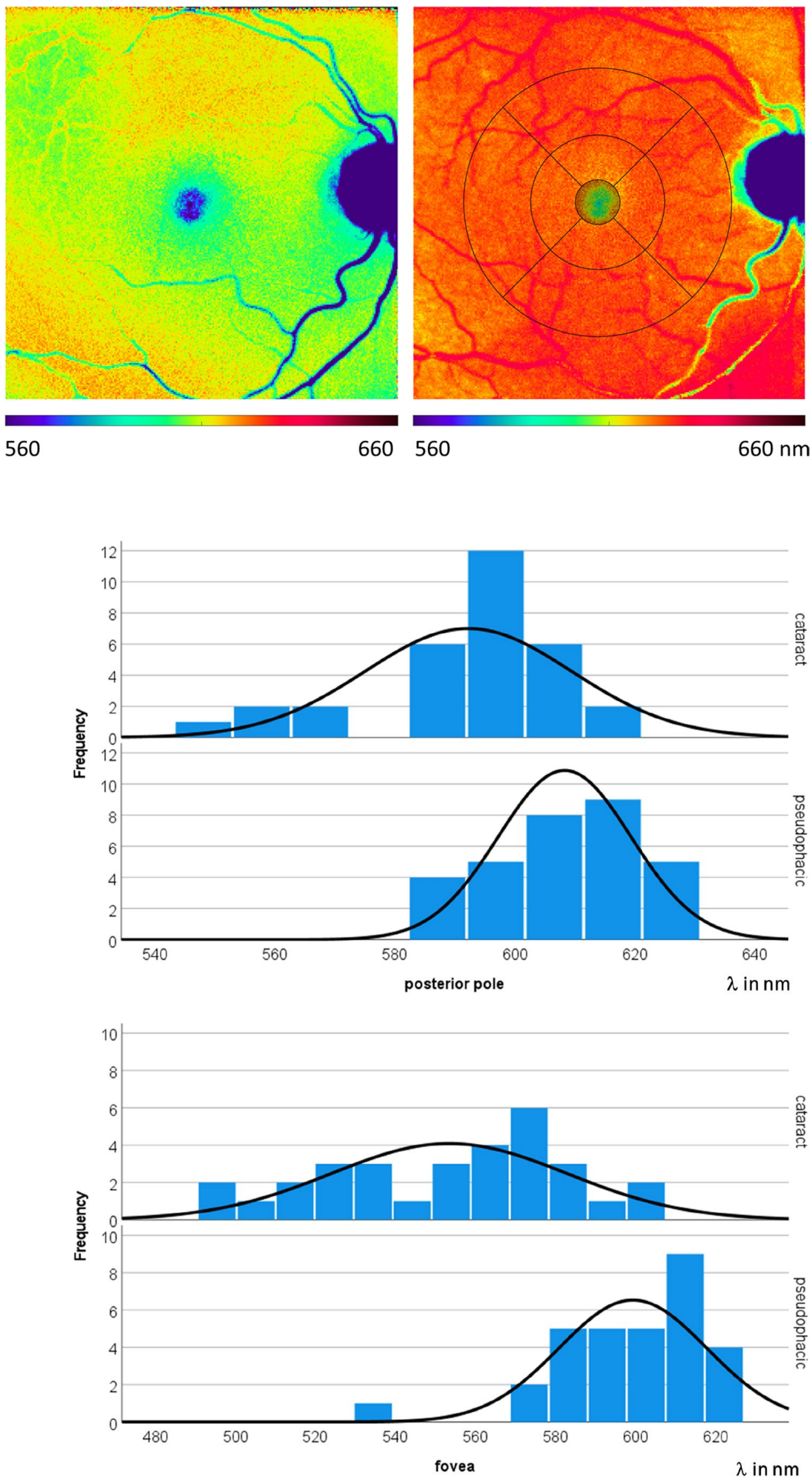
about $515 \mathrm{~nm}$ [5], its removal results in longer emission FAF wavelengths which are in agreement with that of lipofuscin [1]. The difference of PEW pre- and post-surgery is greater in the fovea than at the posterior pole. The reason might be the lower fluorescence intensity due to Xanthophyll absorption of the excitation light. This results in a higher relative contribution of the cataract lens to the total fluorescence in the fovea. In summary, our investigation shows that the fluorescence of the lens shifts the measured FAF toward shorter wavelength. Although this was shown in the current study for cataract lenses only, we assume that this held in general for aged lenses.

Funding Open Access funding enabled and organized by Projekt DEAL.

\section{Declarations}

Conflict of interests None of the authors has conflicting interests and no funding was received for this investigation.

Open Access This article is licensed under a Creative Commons Attribution 4.0 International License, which permits use, sharing, adaptation, distribution and reproduction in any medium or format, as long as you give appropriate credit to the original author(s) and the source, provide a link to the Creative Commons licence, and indicate if changes were made. The images or other third party material in this article are included in the article's Creative Commons licence, unless indicated otherwise in a credit line to the material. If material is not included in the article's Creative Commons licence and your intended use is not permitted by statutory regulation or exceeds the permitted use, you will need to obtain permission directly from the copyright holder. To view a copy of this licence, visit http://creativecommons.org/licenses/by/4.0/.

\section{References}

1. Delori FC, Dorey KC, Staurenghi G, Arend O, Goger DC, Weiter JJ (1995) In vivo fluorescence of the ocular fundus exhibits retinal pigment epithelium lipofuscin characteristics. Invest Ophthalmol 36:718-729

2. Tong Y, Ben Ami T, Hong S, Heintzmann R, Gerig G, Ablonczy Z, Curcio CA, Ach T, Smith RT (2016) Hyperspectral Autofluorescence Imaging of Drusen and Retinal Pigment Epithelium in Donor Eyes with Age-Related Macular Degeneration. Retina 36(Suppl 1):S127-S136. https://doi.org/10.1097/IAE.0000000000001325

3. Schultz R, Klemm M, Meller D, Hammer M (2021) Spectral calibration of fluorescence lifetime imaging ophthalmoscopy. Acta Ophthalmol. https://doi.org/10.1111/aos.14950

4. Brauer JL, Schultz R, Klemm M, Hammer M(2020) Influence of Lens Fluorescence on Fluorescence Lifetime Imaging Ophthalmoscopy (FLIO) Fundus Imaging and Strategies for Its Compensation. Translational vision science \& technology 9:13. https://doi.org/10.1167/tvst.9.8.13

5. Zuclich JA, Previc FH, Novar BJ, Edsall PR (2005) Near-UV/blue lightinduced fluorescence in the human lens: potential interference with visual function. J Biomed Opt 10:44021. https://doi.org/10.1117/1.1990163

Publisher's note Springer Nature remains neutral with regard to jurisdictional claims in published maps and institutional affiliations. 